\title{
Analysis on the Mobile Electronic Commerce Recommendation Model based on the Ant Colony Algorithm
}

\author{
X. ZHANG \& X.P. PANG \\ Hebei University of Economics and Business, Shijiazhuang Hebei, China
}

\begin{abstract}
With the popularity of the internet and the development of e-commerce, the e-commerce system is providing users with more and more choice, and its structure becomes more complex. Users often get lost in a large number of commodity information spaces, and can't find the need goods smoothly. E-commerce recommendatio system can solve the problem. E-commerce recommendation system is the e-commerce sites provide commodity information and advice to customers, sales staff, so that the customers can complete the purchase process simulation software system. The e-commerce recommendation system makes the ecommerce system adapted to the specific needs of each customer, for each user adapted to the user's electronic stores. In the increasingly fierce competition environment, the e-commerce recommendation system in theory and practice has been great development. But with the further expansion of e-commerce systems, the ecommerce recommendation system is facing a series of challenges. In this paper, we will discuss the application of the ant colony algorithm in the mobile electronic commerce recommendation model.
\end{abstract}

KEYWORD: Ant colony algorithm; Mobile e-commerce; Recommend model

\section{INTRODUCTION}

The developed on the basis of Internet, e-commerce is bring profoundly changed the traditional business activities. With the popularity of all kinds of mobile terminal, Internet access is also changing. In addition to continue to use the desktop computer access to the Internet, people began to use the growing popularity of mobile devices to access the Internet, mobile Internet. It is based on the above, in the near future, the application of the mobile Internet will be more diversified, all sorts of new communication mode and commerce mode also begin to enter the life of people, such as mobile chat and mobile positioning, mobile payment, etc. People often use mobile business to describe the new with the help of the mobile communication network social intercourse activity. As an important application of mobile Internet, mobile commerce has attracted many large domestic PC e-commerce giant and operators to enter the mobile e-commerce; they launch and develop the mobile WAP browsing access and all kinds of client applications.

The Era of mobile commerce, the user consumption personalized pursuit, hope enterprise can according to their own characteristics and requirements to provide for their own consumption products and services, without the need for yourself in the vast amounts of information resources by search. Indeed, personalized recommendation is solving the problem of information overload is one of the most effective tools, is also a kind of new marketing tools, to meet the personalized requirements of users, also is advantageous to the enterprise fully tap the potential demand of users, expand sales, improve user satisfaction, and thus enhance the competitiveness of enterprises The Architecture of the Personalized Recommendation System of the E-commerce.

In general, the personalized recommendation system consists of three parts: input module, recommendation algorithm module and output module, including recommendation algorithm is the core of the personalized recommendation system.

\subsection{The input module}

The mainly refers to collect user information record module, including some users of the current and historical information, these information to a certain extent, can reflect the user interest preferences, has the following 7 kinds of main forms:

(1) The user's registration information. In general, users in the site to log in or buying for the first time are required to register, age, gender, etc., which may include users with user interest preference has 
relevance, can be used as the recommended input information.

(2) The implicit browse input. Refers to the user and not trying to search, but can according to user's current information or some kind of behavior analysis, user's interests, in turn, recommend suitable products for customers, including products, browse the path of the user is browsing and users into the product in the shopping cart.

(3) The explicit browse input. This is also the user's browsing behavior as input information recommendation system, with the implicit browse input is relative, the difference is that the user's explicit browse input is purposefully to recommendation system provides information about their own interests and hobbies.

(4) The score input. This is the most common form of input in personalized recommendation system, users can to score of a product, generally divided into 0 to 5 points, system according to the score can be recommended from the Angle of two users: one is the system according to user's score of different products of high and low, that the user interest preference, referring users to its score higher product or similar products; it is to provide a reference for other users.

(5) The evaluation of text input. It refers to the user to output in written form to the evaluation of a certain product. This input usage with grading input similar, the difference is presented in the form of numerical score input, the input text evaluation is presented in the form of text, compare scores, text more rich amount of information, the evaluation content contains more help determine user preferences or provide a reference for other users.

(6) The editors recommend input. It is also a kind of text input, but not to provide this information the user itself, but experts, by the experts in the field of the product reviews of products in the form of text, the purpose of this input for the user to provide more authoritative reference when buying products.

(7) To purchase history. User's purchase history mainly deposited in the background of e-commerce transactions in the database, the user shopping before the detailed records of history, including purchasing time, price, discount, etc.

(8) The keyword input/product attributes. Input refers to the user with the purpose of information search, the products include the keyword search, select or input to find the relevant properties of the product, or the user is browsing product categories as system input, but different from the user's browsing behavior.

\subsection{The recommendation algorithm module}

This module is the core part of the recommendation system; recommendation algorithm performance directly determines the merits of the recommendation system. In this module, the main task to establish the corresponding user model, according to the information input module analysis of user behavior and preferences, and then according to certain way to produce recommendation results.

\subsection{The output function module}

The output module is primarily a recommendation system based on the input information obtained through relevant recommendation algorithm for processing after the recommended to the user, the content of the main forms are:

(1) The output of the related products, which directly to the customer for product recommendations, this is the most widely used a form of output, the output of the current most recommendation systems is to take this form;

(2) The individual evaluation of text output, the other user or relevant experts as the output of evaluation, provide a reference for users to buy the product.

(3) The individual scoring output, the other users' individual score as the output of products;

(4) The average numerical rating output, integrates the other user's score, it is concluded that other users of the product's scoring average;

(5) The email output, including the class most of the e-commerce sites have adopted the way of the output and product recommendations from users will receive regular mail, in this way can keep the contact between the user, so as to reduce customer churn rate. In desktop e-commerce recommendation system input source mainly include two categories: the user information and product project information. Users interested in management by extracting can reflect the characteristics of the User interest information to build the User Profile, including the User's browsing history and for evaluation of product project score or text, etc., to provide recommendation algorithm module to describe the characteristics of the User interest vector, the characteristics of the different recommendation algorithm need different information; Used to organize and manage information resources management at present, the products available for retrieval of project information resources, including the properties of the products, and other products related information, and store it in the Resource in the DB.

\section{THE SUMMARY OF THE ANT COLONY ALGORITHM}

As an important branch of artificial intelligence research, bionic optimization algorithm is to point to by simulating the nature of a certain type of behavior mechanism and applied to the artificial intelligence 
algorithms, mainly includes three types: genetic algorithm (simulated biological natural selection and genetic mechanism), ant colony algorithm (ant colony foraging behavior simulation) and particle swarm algorithm (simulated birds group feeding behavior). Ant colony algorithm is a new kind of intelligent optimization algorithm, by Italian scholars. M first proposed in 1991, its essence is a complex intelligent system, has strong robustness, excellent distributed computing mechanism, and other advanced method combined with the advantages of easily. At present, the ant colony algorithm has become a research hotspot in the field of artificial intelligence, domestic and foreign study of ant colony algorithm has penetrated into multiple applications.

The ant colony algorithm is inspired by nature real ant colony behavior and put forward, based on the ant group in the process of looking for food reflect a series of behavioral characteristics to a random search algorithm of the simulated calculation. In the process of ants foraging, it will release when a path through a special kind of secretion pheromones search path, the ants will depend on the pheromones to communicate, and as the basis of choosing path. When they are in an intersection, will choose a path forward and release a certain amount of pheromone to release the amount of pheromone associated with ants walk path length, the longer the path, the less the pheromone release; When other ants meet again the intersection, the probability of pheromone larger path is selected. Shortest path pheromone, therefore, more and more big, the other is not choose the path of the pheromone will evaporate over time and eventually the whole ant colony will find out the optimal path, namely the optimal solution is obtained. Chart can image shows that the ant search principle.

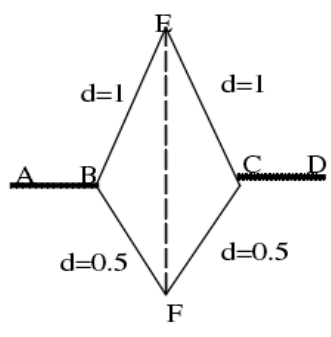

(a)

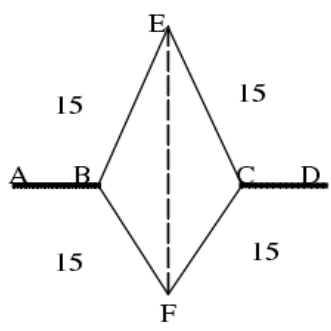

(b)

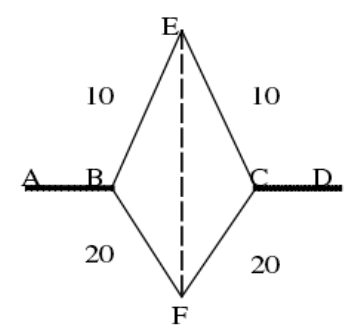

(c)

Figure 1 Ants foraging analog in nature
As shown in figure 1, $\mathrm{A}$ is the nest and $\mathrm{D}$ for food source, in the nest and food source from must be made between $A$ through $\mathrm{E}$ or $\mathrm{F}$ arrive $\mathrm{D}$, or from $\mathrm{D}$ to $\mathrm{A}$, distance $\mathrm{D}$ as shown in figure 1 (A). Assume that each unit of time has 30 ant from $A$ to $D$ or from $\mathrm{D}$ to $\mathrm{A}$, after each path pheromone to 1. Initial moment, BF, FC, BE, EC are no information exists, namely the pheromone is 0 , the ants in $\mathrm{A}$ and $\mathrm{D}$ can $\mathrm{BE}$ selected at random path, from the perspective of statistics can think each path choice probability is the same, as shown in figure 1 (b). After a unit of time, in the path of the pheromones are on the path landing the BEC twice, so there will be 20 ant choose D after landing the path to food. Over time, more and more ants will be in the probability of landing the path choice, finally find the optimal path of landing. The following simple introduced a mathematical model of basic ant colony algorithm to construct:

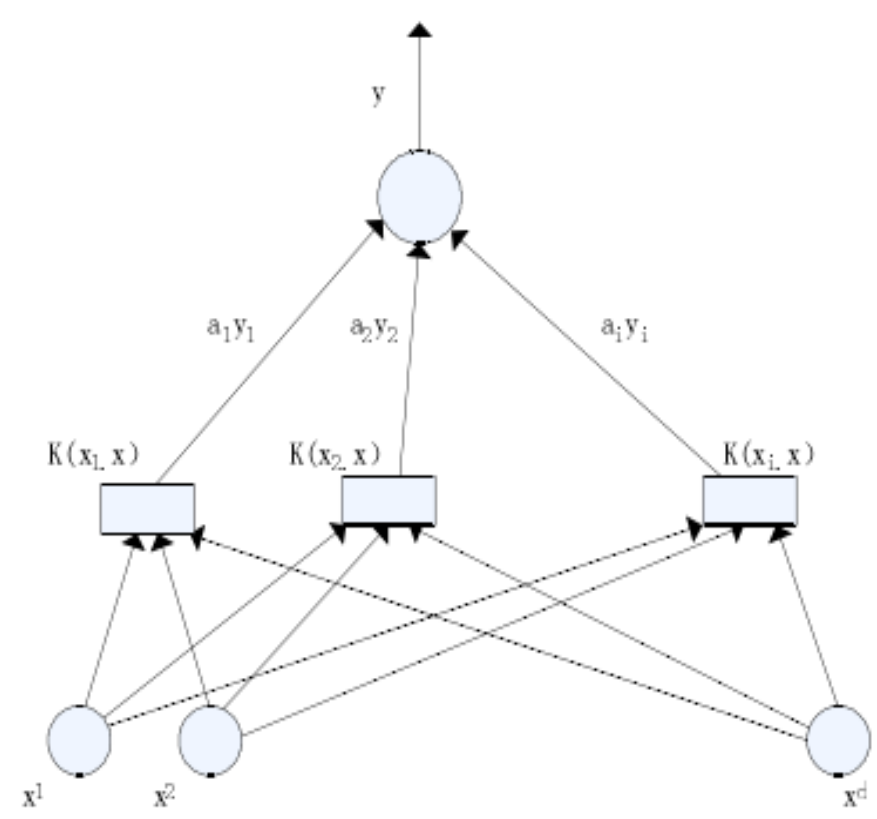

Figure 2 Support vector machine (SVM) model

First we introduce the following parameters: Assuming the collection $C=\left\{C_{1}, C_{2}, \cdots C_{n}\right\}$ is a collection of nodes, the all paths gather $L=\left\{l_{i j} \mid c_{i}, c_{j} \subset C\right\}$ is a collection of nodes in two connections in $C, b_{i}(t)$ show the number of ants in node $i$ in moment $t, \tau_{i j}(t)$ is a pheromone in $(i, j)_{\text {in moment }} t, m_{\text {is }}$ the total number of the ant colony of ants, $\Gamma$ is the collection. The definition is: $m=\sum_{i=1}^{n} b_{i}(t), \Gamma=\left\{\tau_{i j}(t) \mid c_{i}, c_{j} \subset C\right\}$

The Ant - Cycle model, the Ant after completing a Cycle for all update the pheromone trails, USES a global update strategy; In the Ant - Quantity model, 
Ant - Density model is adopted in the local update strategy, ants every step, is to update the pheromone on the path. Compared with local update strategy, global pheromone update can improve the efficiency of search, because in the ant colony algorithm, only constitute the edge of the global optimal path can increase the pheromone level, the other side of the pheromones might volatilization will reduce gradually, after several search, belong to the best path pheromone levels will be significantly higher han the other path so as to improve the efficiency of search.

\section{THE APPLICATION OF THE ANT COLONY ALGORITHM IN THE MOBILE ELECTRONIC COMMERCE RECOMMENDATION MODEL}

The electronic commerce development cannot leave the network user's supervision and evaluation, the development of China's e-commerce is still in the primary stage, some netizens for not familiar with electronic commerce, online shopping credibility is not high, the evaluators of the authority of evaluation data has a question, these affect the validity of the evaluation. However in e-commerce for customers recommend, evaluation and essential goods, by building a model of mobile electronic commerce recommendation based on ant colony algorithm, and effectively solve the data sparseness, user evaluation data by constructing classification discriminate function at the same time, through the classification and evaluation of the usefulness of classifier and score, give evaluation usefulness ranking and recommend to the customers, established a relatively effective e-commerce personalized recommender system model.

Based on desktop electronic commerce recommendation system architecture, combined with the particularity of mobile commerce, introducing the user real-time information, put forward a kind of personalized recommendation system based on mobile commerce application architecture. According to the former in the face of recommended source of input data analysis, User information can be broken up into history and User interest information User preferences information two modules, and respectively for storage: User history interest management by extracting can reflect the characteristics of the User information of historical interest in building the User historical Profile, mainly includes the User's browsing history and for evaluation of product project score or text, etc., in the mobile commerce in order to avoid repeated recommendation, improve the efficiency of recommendation, can also join the User has recommended projects, provide recommendation algorithm module with the characteristics of the history of User interest vector. Real-time User preferences management can reflect the current User preference to build User Timely Profile, mainly including the User's current of the time, location, weather, etc., to provide recommendation algorithm module described users interested in real-time feature vector; Used to organize and manage the information resources management project of retrievable information resources, including the properties of the products, and other products related information, and store it in the Resource in the DB.

\section{CONCLUSIONS}

The mobile commerce as a new type of electronic commerce, has its own particularity, for the personalized recommendation systems they are also put forward higher request, cannot copy desktop ebusiness environment or traditional personalized recommendation algorithm system. In the mobile commerce environment, to establish a more reasonable, high efficient and real-time personalized recommendation system has become a very important problem in the field of mobile commerce. The ant colony algorithm is widely used in recent years the bionic optimization algorithm, the ant colony algorithm was used in the mobile commerce personalized recommendation system structure, is able to eliminate redundant information in the vast amounts of information, thus it can greatly improve the speed and effectiveness of information retrieval.

\section{REFERENCES}

[1] Ya Zhang,Weiyuan Chen, Zibin Yin. Collaborative filtering with social regularization for TV program recommendation. Knowledge-Based Systems. 2013(10).

[2] Supreet Reddy Mandala, Soundar R. T. Kumara, Calyampudi Radhakrishna Rao,Reka Albert. Clustering social networks using ant colony optimization. Operational Research. 2013 (1)

[3] Janusz Sobecki. Ant Colony Metaphor Applied in User Interface Recommendation. New Generation Computing. 2012(3).

[4] Julia Handl, Bernd Meyer. Ant-based and swarm-based clustering. Swarm Intelligence. 2013 (2).

[5] Hanene Azzag, Gilles Venturini, Antoine Oliver, Christiane Guinot. A hierarchical ant based clustering algorithm and its use in three real-world applications. European Journal of Operational Research. 2013 (3).

[6] David Goldberg, David Nichols, Brian M. Oki, Douglas Terry. Using collaborative filtering to weave an information tapestry. Communications of the ACM. 2013 (12).

[7] Badrul Munir Sarwar. Sparsity, scalability, and distribution in recommender systems. 2012(8).

[8] A. Colorni, M. Dorigo, V. Maniezzo. Distributed optimization by ant colonies. Proceedings of the First European Conference on Artificial Life. 2013(8).

[9] Schafer J B, Konstan J, Riedl J. Recommender Systems in ECommerce. ACM Conference on Electronic Commerce (EC99). 2012(6).

[10] Marco Dorigo, Gianni Di Caro. Ant algorithms for discrete optimization. Artificial Life. 2012(6). 